\title{
Téoros
}

Revue de recherche en tourisme

\section{L'union fait la force : les musées découvrent le potentiel du réseau}

\section{Sylvie Gagnon}

Volume 11, numéro 2, juillet 1992

Quand les musées s’ouvrent au tourisme...

URI : https://id.erudit.org/iderudit/1078091ar

DOI : https://doi.org/10.7202/1078091ar

Aller au sommaire du numéro

Éditeur(s)

Université du Québec à Montréal

ISSN

0712-8657 (imprimé)

1923-2705 (numérique)

Découvrir la revue

Citer cet article

Gagnon, S. (1992). L’union fait la force : les musées découvrent le potentiel du réseau. Téoros, 11(2), 39-40. https://doi.org/10.7202/1078091ar d'utilisation que vous pouvez consulter en ligne.

https://apropos.erudit.org/fr/usagers/politique-dutilisation/ 


\title{
L'union fait la force: les musées découvrent le potentiel du réseau
}

\author{
Sylvie Gagnon*
}

$\mathrm{L}$ 'importance des institutions museales dans l'infrastructure touristique est indéniable. En attirant des visiteurs, le musée, quelle que soit la nature de ses collections, contribue de plus en plus à l'essor du tourisme à l'échelle locale et régionale. Le musće est une manifestation de l'identité culturelle de lacommunautédontilestissu.Le touristes"y rend pour prendre contact avecl' histoire et la culture qui ont façonné la région hôte.

Les expositions grand public - les blockbusters - ont une incidence directe sur le développement touristique, comme en témoigne, au cours des dernières années, l'énorme succès des expositions Van Gogh et Rembrandt au Rijksmuseum a Amsterdam. C'est parfois l'institution muséale en soi qui constitue l'attrait touristique. Au Ontario Science Centre a Toronto, par exemple, seulement un tiers des visiteurs proviennent de Toronto et de l'Ontario; les autres deux tiers sont composés de clientèles touristiques en provenance d"autres provinces et pays. On prévoit que le Biodôme, par son statut unique, connaîtua le même genre de succes.

\section{La reconnaissance par le milieu touristique}

Des gestes de reconnaissance de la part du milieu touristique quant à l'impact des institutions muséviles émergent de toute part. Au Québec, les associations touristiques régionales ont décemé 25 prix à nos musćes depuis 1990.

En Europe, grâce au parrainage de la Commission des Communautés européennes et dañs le cadre de l'Année européenne du Tourisme, le concours Tournusé est lancé pour la première fois en mai 1991. Quelque 4500 musces et 1500 professionnels du tourisme y participent. Se donnant pour thème Tourisme et Musées, le concours a pour objet de sensibiliser le grand public et les professionnels à l'importance du rôle cconomique du musée.

\footnotetext{
Madame Sylvie Gagnon est directrice de la Société des musées québécois.
}

Le grand prix de Tourmusé a été décerné à une institution qui s'apparente en popularité au Ontario Science Centre. Ouvert en septembre 1988, le Musée de l'image animée (MOMI) raconte l'histoire magique du cinéma etde la télévision. Le MOMI fait partie des dix centres de loisirs les plus visités de Londres et constitue un attrait qui n'a pas d'equivalent au monde.

Les deux autres prix - ceux de l'excellence régionale et đe l'originalité — ont été attribués en reconnaissance de la promotion d'un réseau d'institutions muséales.

Le prix de l'excellence régionale a été décerné au Musée du vin de Torgiano, en Italie, pour un projet monté en collaboration avec un hôtel de la région. Il s'agissait d'un circuit de dix jours permettant à des groupes de 25 personnes de découvrir les musées agricoles, les richesses artistiques et les métiers d'artde larégion, le couur ducircuit étant le Musćedu vin.

Le prix de l'originalitế a été remis à Action Inter-Musées qui a conçu la Carte des Musées et Monuments de la région parisienne. Crúce en 1988, la Carte est un laissez-passer valable pour une, trois ou cinq journées consécutives, donnant accès aux collections permanentes de 64 musćes et monuments de la région. La Carte s"adresse tout particulièrement aux visiteurs et touristes de passage à Paris,

\section{Les regroupements et leur promotion}

La promotion de réseaux d'institutions muséales, plutôt que celle d'institutions isolées, constitue une tendance récente et fort populaire.

En 1990, la Société des musées québécois devait réaliser une campagne de promotion des musćes de l'île de Montréal. Pour ce faire, elle a d'abord effectué une étude ${ }^{(1)}$ sur la question de la promotion coopérative: à cetteépoque, la promotion de regroupements muséaux était rare. Il a fallu se rendre à San Diego, au Delaware, à Boston etă New York pour trouver les modẻles recherchés.

Deux ans plus tard, la situation n'est plus du tout la même. Presque tous les regroupements régionaux du Québec offrent soit un passeport, soit un circuit, soit des outils promotionnels conjoints. Certains faits expliquent ce phếnomène : une clientèle commune, une force de frappe indéniable et des conditions de réussite réunies,

\section{Une clientèle commune}

Le visiteur d'un musée prendra probablement plaisir à en visiter d'autres, on le sait. Voila pourquoi les musces se perçoivent comme étant complémentaires plutôt que concurrentiels. Les études démontrent que le profil socio-démographique des visiteurs est

Tableau 1

Profil des visiteurs de museels

\begin{tabular}{|c|c|c|c|}
\hline $\begin{array}{l}\text { Art Gallery } \\
\text { of Ontario }\end{array}$ & $\begin{array}{l}\text { Royal Ontario } \\
\text { Museum }\end{array}$ & $\begin{array}{l}\text { Ontario Science } \\
\text { Centre }\end{array}$ & $\begin{array}{l}\text { Metropolitain } \\
\text { Toronto Zoo }\end{array}$ \\
\hline $2 \%$ enfants & $14 \%$ enfants & $20 \%$ enfants & $27 \%$ enfants \\
\hline plus âgés & $\Leftrightarrow$ & $\Longrightarrow$ & moins âgés \\
\hline plus scolarisés & $\Leftrightarrow$ & $\therefore a$ & moins scolarisés \\
\hline plus de fenmes & $\Leftrightarrow$ & 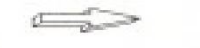 & plus d'hommes \\
\hline visitcurs sculs & $\Leftrightarrow$ & $\Rightarrow$ & visiteurs en groupe \\
\hline Écoutent moins la tv & $\Leftrightarrow$ & $\Longrightarrow$ & cooutent plus la tv \\
\hline $\begin{array}{l}\text { passent le plus des } \\
\text { femps it lire }\end{array}$ & $\Rightarrow$ & $\Leftrightarrow$ & $\begin{array}{l}\text { consacrent moins de } \\
\text { temps a la lecture et plus aux jeux }\end{array}$ \\
\hline $\begin{array}{l}\text { vont le plus souvent } \\
\text { au cinenta }\end{array}$ & $\Longrightarrow$ & $\Leftrightarrow$ & $\begin{array}{l}\text { vont le moins souvent } \\
\text { au cincma }\end{array}$ \\
\hline
\end{tabular}




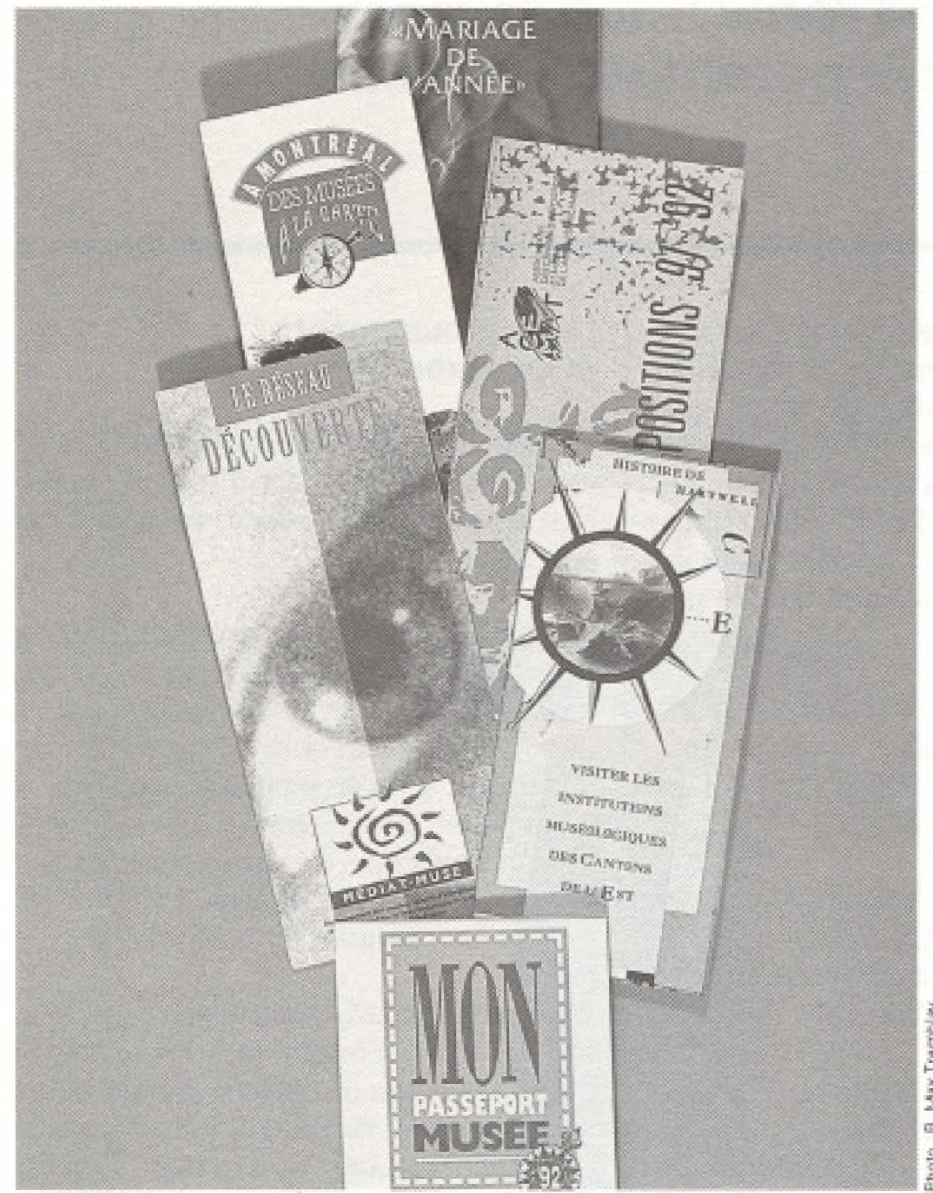

Les musées québécoisant réaliséces brochunes au cours de la dernière annére : elles rémoigneni d'un engagement dans la diffusion aupres d'wne clientelle touristique d̀ exploiter.

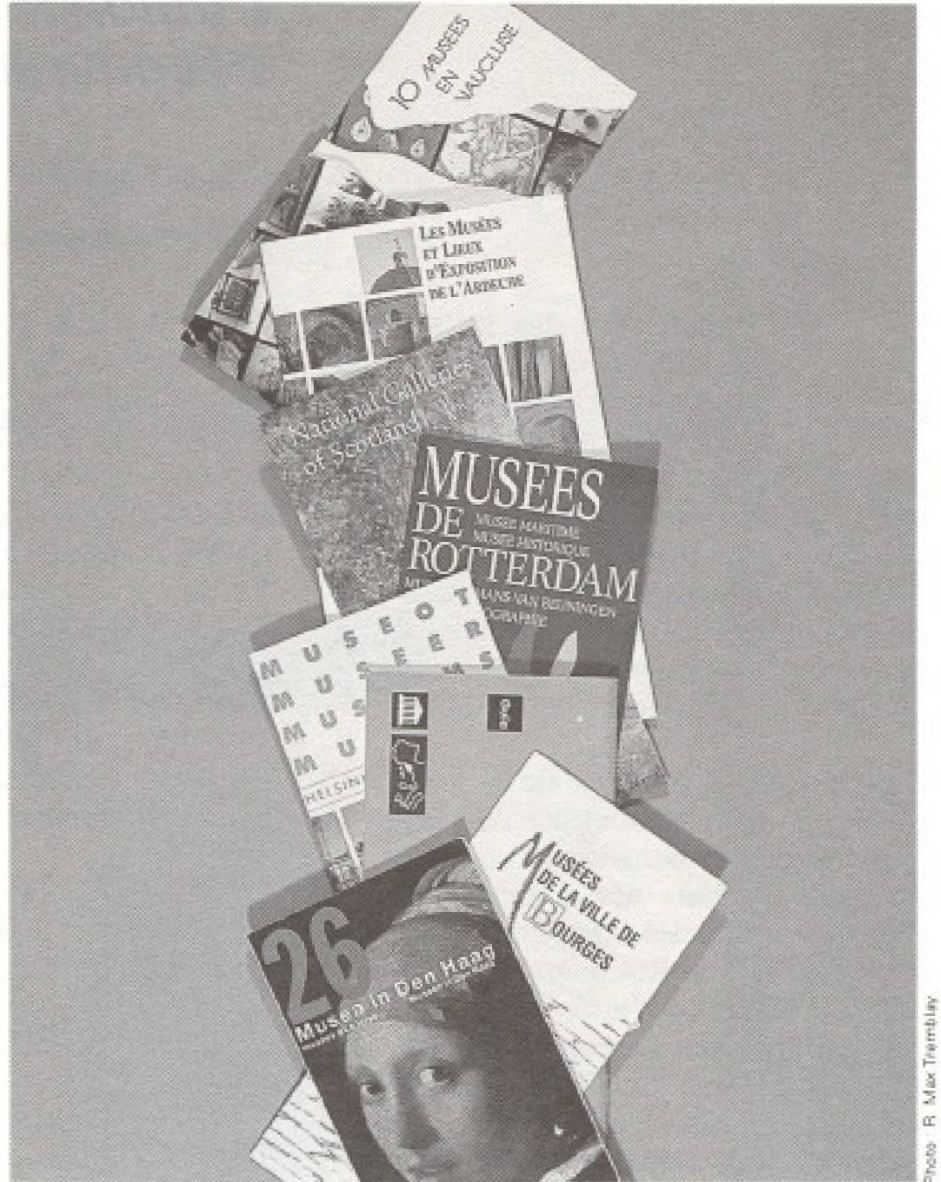

L'Europe et ses mustes dicouvrent le potentiel d wene pramotion en réseau. semblable dans tous les musées du monde. Une des premières synthèses des études de clientèle, faiteen $1978^{\text {(2h }}$ dresse le portrait du visiteur-type en se basant sur 270 études de clientèle de musées d'art. Selon ses résultats, il y a une forte concentration des visiteurs entre 18 et 44 ans, surtout de sexe féminin (57\%), des professionnels ou cols blancs ayant un revenu supérieur à la moyenne.

Par ailleurs, en 1990, quatre institutions de Toronto-l'Ant Galleryof Ontario, le Royal Ontario Museum, I'Ontario Science Centre et le Metropolitan Toronto Zoo - se sont réunies dans le but d'étudier non seulement les similitudes de leurs clientèles mais Egalement les différences. L'enquête, dont les principaux résultats sont présentés dans le tableau 1 ci-dessus, révèle que le zoo, bien qu'il y ait plusieurs ressemblances chez la clientèle, attire un public plus diversifié que la galerie d'art.

\section{Une force de frappe}

En se regroupant pour commercialiser leurs produits, les institutions muséales réalisent des économies, augmentent leur visibilité et profitent de plus vastes possibilités de promotion. Ensemble, elles peuvent offrir un produit culturel susceptible d'attirer une clientèle plus nombreuse et, ce faisant, obtenir l'appui de commanditaires et de gouvernements. Parailleurs, les associations touristiques régionales encouragent ce type de promotion et ont mis au point des politiques à cet égard.

\section{Les conditions de succès}

Le succès d'une promotion issue d'un effort collectif dépend de certaines conditions. Premièrement, l' impact d'une promotion est plus marqué si les institutions en question sont situées sur un territoire restreint. Aussi, la qualité du leadership et de l'équipe coordonnant le projet ainsi que la confiance mutuelle sont essentielles â la rếussite de l'intervention. Finalement, il semble qu' un produit réunissant des institutions différentes - depuis le musée d'art jusqu'au zoo, en passant par les lieux historiques - ait plus de chances de réussite.

\section{Les retombées}

A l'heure actuelle, les actions promotionnelles des regroupements semblent donner lieu à des résultats concluants. Il existe toutefois très peu de documentation sur l'évaluation de la promotion conjointe d'institutions muséales et sur ses retombées touristiques. Si la collaboration entre le milieu musćal et l'industrie du tourisme semble fructueuse, il est à espérer qu'elle puisse s'étendre au domaine de la recherche, donnant ainsi aux institutions muséales et touristiques des mesures quant à leur influence réciproque. $f$

\section{BIBLIOGRAPHIE}

(4) SOCIÉTÉ DES MUSÉES QUÉBECCOIS, R日cherche et analyse on vue de P'blaboration d'un plan de promotion des petites et moyennes plan de promotion des pentites ét môyennens

ta des musées québécois, 1990 . Michael and Paul BROWN. Audience Studies of the Performing Arts and Museums. A Critical Review Nationg Endowment for the Ants flesearch Division 1978 Endowment or the Ans, hesearch Division, 1978 ART Gallery OF ONTARIO, ROYAL ONTARID MUSEUM, ONTARIO SCIENCE CENTRE. Metropolitan TORONTO ZOO, Audience Research Consortium. Summary of the First Year Rasearch, Toronto, Ernst \& Young, 1990, p.11 